\title{
Simulation of pedestrian evacuation with the obstacles based on a dynamic cellular automaton model
}

\author{
Nuo Zhu ${ }^{1, \text { a }}$, Peiying Yang ${ }^{1, b}$ and Xiang $\mathrm{Li}^{1, \mathrm{c}}$ \\ ${ }^{1}$ Information center of CAAC, 155\# Dongsi Xidajie, Beijing, 100710, China \\ a zhunu051@163.com, bpy_yang@caac.gov.cn, ${ }^{\mathrm{c}}$ lixiang@caac.gov.cn
}

Keywords: cellular automata, pedestrian evacuation, evacuation time.

Abstract: Simulation of pedestrian evacuation flow in a room with fixed obstacles is presented based on cellular automata (CA) in this paper. Pedestrian moving rules are established, according to two-dimensional cellular automaton Moore neighborhood. The dynamic parameters are formulated to simplify tactically the decision-making process of pedestrians, which can reflect the pedestrian judgment on the surrounding conditions. We propose the improved Dijkstra metric to compute the values of static floor field for evacuation system with obstacles based on the Euclidean distance. The simulation results of the improved and previous model are compared and analyzed. Simulation results show that evacuation time decreases nonlinearly when exit width is increased, there is a linear relationship between evacuation time and pedestrian density. The simulation results correspond with the actual, it is instructional significant for pedestrian evacuation.

\section{Introduction}

Cellular automata (CA) model has been adopted widely and well received as an effective tool in the study of this sphere [1], a real-coded cellular automata [2] is proposed to simulate the pedestrian dynamics in evacuation. The evacuation process with obstacles is simulated using lattice gas model. A floor field model [3] was modified to simulate pedestrian evacuation flow with internal obstacles and multi-exit. Dynamic Parameters Model [4-9] has been used to simulate and study the pedestrian flow. An improved dynamic parameter model [10] is presented, which reflects the judgment of pedestrians on surrounding conditions and the action of choosing or decision. It is well accepted that rational pedestrians are intelligent and adaptive to the dynamic conditions around by constantly seeking and choosing an optimum route. So pedestrians intend to leave room within the shortest possible time, will try to reduce the evacuation imbalance caused by obstacles. However, the model [10] has not been to taken into account the impact of obstacles on pedestrian evacuation, the obstacles will have an impact on pedestrians' evacuation routes and evacuation time, which is essential for pedestrian safety. In this paper, the DPM with obstacles based on model [10] is presented to simulate the decision-making process of pedestrians, considering the effects of obstacles and jam around exits.

\section{Model description}

The model is defined on a discrete $(W+2) *(W+2)$ cell grid in the two-dimensional system, pedestrian cannot pass through obstacles and must leave evacuation system through exits. After leaving system, pedestrian will not reenter the room. In each discrete time step, pedestrians choose to wait or move to the next possible position in the movement field (see Fig. 1(b)). A $3 * 3$ matrix of transition payoff (see Fig. 1(c)) is constructed. 


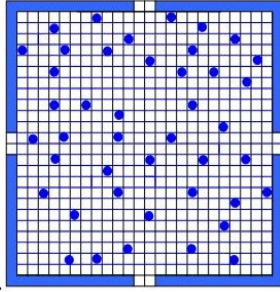

(a) Simulation system

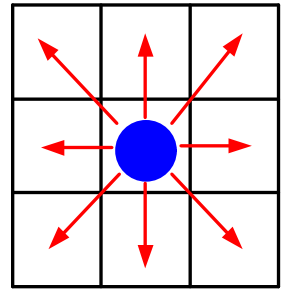

(b)The movement field.

\begin{tabular}{|l|l|l|}
\hline $\mathbf{P}_{-1 ; 1}$ & $\mathbf{P}_{-10}$ & $\mathbf{P}_{-11}$ \\
\hline $\mathbf{P}_{0 ; 1}$ & $\mathbf{P}_{0,0}$ & $\mathbf{P}_{0,1}$ \\
\hline $\mathbf{P}_{1 ; 1}$ & $\mathbf{P}_{1,0}$ & $\mathbf{P}_{1,1}$ \\
\hline
\end{tabular}

(c)The matrix of transition payoffs

Figure 1 Simulation model of evacuation pedestrian

Direction-parameter: Once the geometry of the room is determined, each cell is assigned a constant value representing its distance to the exit. The smaller distance, the greater attractiveness it has. However, model assumes pedestrians cannot pass through obstacles, so pedestrians must reach destination detour or avoid obstacles. We propose the improved Dijkstra metric to compute the values of static floor field based on the Euclidean distance. We define the cells around obstacles which pedestrians must pass through as hinge cells, the direction will change while imaginary flow via hinge cells. There is an hierarchical relationship between the hinge cells, exits are defined as the first-class hinge cells, imaginary flow following pass through second-class, third-class, $\ldots, i$ th-class hinge cells, the $i$ th-class hinge cells are the parent of the (i+1)th-class hinge cells and the offspring of the (i-1)th-class hinge cells. Every cell has parent hinge cells and all parent hinge cells attributes to exits, the value of static floor field is the sum of straight-line Euclidean distance between parent hinge cells.

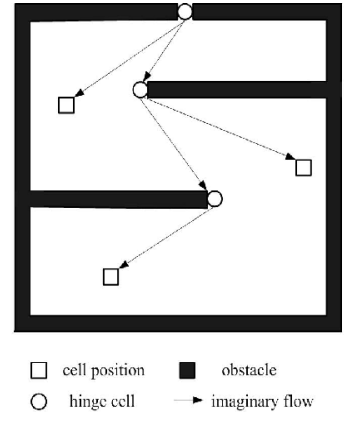

(a) The evacuation route chain.

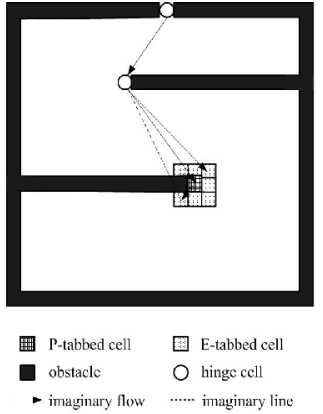

(b) The Expanding of imaginary flow

Figure 2. Pedestrian evacuation route chain and the imaginary flow Expanding from P-Tabbed cell to E-Tabbed cell.

$$
D_{i j}=\frac{S_{00}-S_{i j}}{\sqrt{i^{2}+j^{2}}}
$$

Where $S_{00}$ - the shortest distance from the core cell in the movement field to the evacuation exits. ${ }_{i j}$

- the shortest distance from the cell $(\mathrm{i}, \mathrm{j})$ in the movement field to the evacuation exits

Empty-parameter indicates whether the cell is occupied or not. The values of elements of Empty-parameter matrix are given as

$$
E_{i j}=\left\{\begin{array}{lr}
\max \left(D_{i j}\right), & \text { empty cells, } \\
0, & \text { the core cell }, \\
-\max \left(D_{i j}\right), & \text { occupy cells }
\end{array}\right.
$$

Cognition-parameter describes the effects of pedestrian jam around exits and the width of exits. The values of elements of Cognition-parameter matrix are given as:

$$
C_{i j}=\max \left(\max _{m}\left(\frac{d_{m} \cdot\left(p_{\mathrm{in}}^{t} / S_{i n}^{\mathrm{t}}\right.}{d_{L} \cdot\left(p_{m}^{t} / S_{m}^{\mathrm{t}}\right)}\right)\right)
$$

Where $d_{m}$-the width of m-th exits; ${ }^{d_{L}}$-the sum width of exits; $p_{\text {in }}^{t}$-the number of pedestrians in the system at t time step; $p_{\mathrm{m}}^{t}$-the number of pedestrians around m-th exits at t time step; $S_{\text {in }}^{\mathrm{t}}$-the region of the evacuation system at t time step; $S_{m}^{\mathrm{t}}$ - evacuation region around $\mathrm{m}$-th exits at t time step . 


\section{Rules}

(1) In the model, the pedestrian can only move one cell and has nine possible target positions to select in each time step.

(2) Define transition payoff $\mathrm{P}_{i j}$ as:

$$
\mathrm{P}_{\mathrm{ij}}=\alpha \mathrm{D}_{\mathrm{ij}}+\beta \mathrm{E}_{\mathrm{ij}}+\gamma \mathrm{C}_{\mathrm{ij}}
$$

$\alpha+\beta+\gamma=1,(\alpha, \beta, \gamma \geq 0)$, in every time step, the pedestrian would choose the cell with the largest value $\mathrm{P}_{M} \quad\left(\mathrm{P}_{M}=\max \left(\mathrm{P}_{i j}\right)\right)$ in the matrix of transition payoff as his or her target position.

(3) If more than one possible target cells rank the highest in value ${ }^{P_{i j}}$, only one of them will be chosen as the target position randomly with equal probability.

(4) A conflict occurs when any two or more pedestrians attempt to move to the same target position. In this situation, only one of them will be chosen randomly with equal probability.

(5) When a pedestrian moves into the exit cell, at the next time step the pedestrian will leave the room. After leaving the room, the pedestrian would not reenter the room.

(6) When all pedestrians leave the room and there is no pedestrian in the evacuation room, the simulation procedure is terminated.

\section{Simulation analysis}

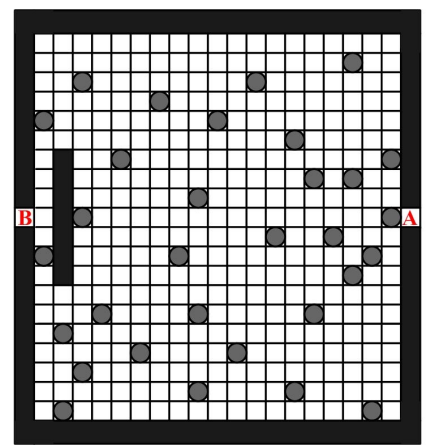

(a) evacuation environment

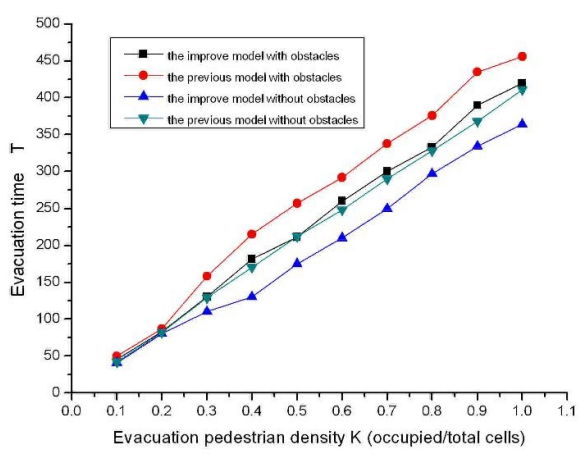

(b) result of comparison

Fig. 3. The comparison between previous and improve model with (and without) obstacles when $\mathrm{W}=20, \mathrm{~L}=1$.

In order to show the advanced nature of our model, we compare it with the previous model [42] in the same conditions. $\mathrm{W}=20, \mathrm{~L}=1, \delta_{E}=0.6, \delta_{H}=0.4, \alpha=0.2, \beta=0.6, \gamma=0.2$. As shown in Fig. 3, we plot the evacuation time $T$ versus pedestrian density $K$ with respect to the previous model and our model. When pedestrian density $K<0.3$, pedestrians could have their own activity freely when low-density, there is few congestion, so the evacuation time almost no difference between previous models and our model. However, with the pedestrian density gradually increasing ( $K \geq 0.3$ ), the mutual interference between pedestrians is much more than low-density, pedestrians' movement become difficult under this condition. Since Cognition-parameter is considered in our model, when the exit which pedestrians firstly choose is too crowded, pedestrians will change their idea and move towards to another exit (or the hinge cell) after several time steps. It has a positive effect of the evacuation, thus our model shows clear superiority than previous model. 


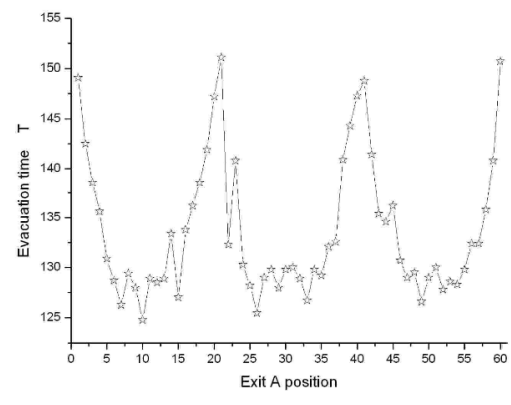

(a) without obstacles

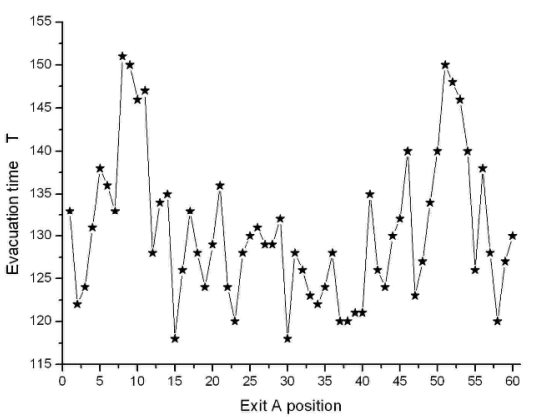

(b) with obstacles

Fig. 4. the evacuation time for evacuation system for different exit A positions when $W=20, L=1, K=0.3$

Fig. 4 shows the evacuation time for evacuation system without obstacles and with obstacles for different exit A positions. As shown in Fig. 4 (a), the evacuation time presents "W" with the increasing of exit positions and the minimum values often appear at the center of the walls, such as 10, 25, and 50. As shown in Fig. 4 (b), the evacuation time shows irregular change with the increasing of exit positions, the minimum values appear at 15,30 , and 57 . Since the existence of obstacles in the evacuation system interfere pedestrians' evacuation trajectory, so with the changing of exit positions, a corresponding change in evacuation route. By comparison, we find that two curves have the same minimum value range at 25-35. When the symmetry of exit layout is taken into account, we recommend that the optimum position of exit $\mathrm{A}$ is 30 .

\section{Conclusions}

In this paper, simulation of pedestrian evacuation with fixed obstacles is presented based on cellular automata (CA). A simple scaling law is proposed to model the dependence of evacuation time with the number of persons, the obstacles and exit width, according to two-dimensional cellular automaton Moore neighborhood. The dynamic parameters are formulated to simplify tactically the decision-making process of pedestrians, considering the effects of pedestrian jam around exits (or the hinge cells) and the width of exits on evacuation path selection in order to reduce evacuation imbalance caused by the exits and obstacles layout, which can decide the pedestrian's choice of action. The simulation results of the improved and previous models are compared and analyzed. The relationships of evacuation time versus pedestrian density and exit width are studied. Simulation results show that evacuation time decreases nonlinearly (negative exponential distribution) when exit width is increased, there is a linear relationship between evacuation time and pedestrian density. The simulation results correspond with the actual, it is instructional significant for pedestrian evacuation.

\section{Acknowledgements}

This paper is financially supported by the National Natural Science Foundation of China (Nos. 11422221 and 11672289).

\section{References}

[1] C. Burstedde, K. Klauck, A. Schadschneider, J. Zittartz, Simulation of pedestrian dynamics using a two-dimensional cellular automaton, Physica A 295 (2001) 507-525.

[2] D. Helbing, P. Molnar, Social force model for pedestrian dynamics, Phys. Rev.E 51 (5) (1995) 4282-4286.

[3] G. Antonini, M. Bierlaire, M.Weber, Discrete choice models of pedestrian walking behavior, Transp. Res. Part B 40 (2006) 667-687.. 
[4] Th. Robin, G. Antonini, M. Bierlaire, J. Cruz, Specification, estimation and validation of a pedestrian walking behavior model, Transp. Res. Part B 43 (2009) 36-56.

[5] H. Yue, H. Hao, X. Chen, C. Shao, Simulation of pedestrian flow on square lattice based on cellular automata model, Physica A 384 (2007) 567-588.

[6] H. Yue, C. Shao, Z. Yao, Pedestrian evacuation flow simulation based on cellular automata, Acta Phys. Sinica 58 (2009) 4523-4530.

[7] H. Yue, C. Shao, X. Chen, H. Hao, Simulation of bi-directional pedestrian flow based on cellular automata model, Acta Phys. Sinica 57 (2008) 6901-6908.

[8] H. Yue, Zhang B Y, Shao C F, Xing Y, Exit selection strategy in pedestrian evacuation simulation with multi-exits, Chin. Phys. B 23(2014) 050512.

[9] Zhang L, Yue H, Li M, Wang S, Mi X Y, Simulation of pedestrian push-force in evacuation with congestion, Acta Phys. Sin.64(2015) 060505 (in Chinese).

[10] Zhu Nuo, Jia Bin, Shao Chun-fu, Yue Hao. Simulation of pedestrian evacuation based on an improved dynamic parameter model, Chinese Physics B.21 (5) (2012). 050501. 